\title{
Expression of microRNAs-21 and-223 in hepatocellular carcinoma in hepatitis C virus infected Egyptian population
}

\author{
Eman A. Elghoroury ${ }^{1}$, Soheir A. Abdel Maksoud ${ }^{1}$, Dina Kandil ${ }^{1}$, Mona Raafat El Kafoury ${ }^{1}$, Eman Mahmoud Hassan, \\ Eman Awadallah ${ }^{1}$, Mohamed A. Hussein ${ }^{2}$, Hany Ahmed Elghobary ${ }^{3}$ \\ ${ }^{1}$ Clinical and Chemical Pathology Department, National Research Centre, El-Buhouth St., Dokki, Cairo, Egypt. \\ ${ }^{2}$ Internal Medicine Department, Cairo University, Cairo, Egypt. \\ ${ }^{3}$ Clinical and Chemical Pathology Department, Cairo university, Cairo, Egypt.
}

\section{ARTICLE INFO \\ Article history: \\ Received on: 02/04/2017 \\ Accepted on: 05/08/2017 \\ Available online: 30/09/2017}

Key words:

CAP2, HCV, HCC, MIF, microRNAs.

\begin{abstract}
Background: The prognosis of hepatocellular carcinoma (HCC) in Egypt is poor due to high prevalence of hepatitis $\mathrm{C}$ virus (HCV). Moreover, the performance of laboratory markers for diagnosis of HCC at early stages has not been optimal.

Objective: We aim to assess expression of miR -21 and miR-223 in HCC patients in the presence of HCV infection and correlate this expression with other clinical, laboratory features of HCC and other markers such as cyclase-associated protein2 (CAP2) and macrophage migration inhibitory factor (MIF).

Subjects and Methods: The study included 40 cirrhotic, $40 \mathrm{HCC}$ patients and 40 healthy subjects. Assessment of miRNAs was done using RT-PCR (Applied Biosystems).

Results: Levels of miR-21 were higher in patient groups compared to control group and in HCC patients compared to other patients ( $\mathrm{p}=0.001,0.002$ respectively). Serum levels of miR-223 showed no significant differences between cirrhotic patients and control group $(\mathrm{p}=0.2)$, while marked decrease in miR-223 expression levels was found in HCC patients compared to cirrhotic patients and control group ( $\mathrm{p}=0.004,0.001$ respectively). Conclusion: Altered serum miR-21 and -223 may play role in pathogenesis of HCC in the presence of HCV infection and might serve as a potential biomarker for detection of early HCC.
\end{abstract}

\section{INTRODUCTION}

Egypt has the highest hepatitis $\mathrm{C}$ virus (HCV) prevalence worldwide (Elgharably et al., 2017). Thus, HCV is considered a major public health problem in Egypt. Complications of $\mathrm{HCV}$ infection in Egypt are responsible for $67 \%$ of morbidity related to liver disease (Atta et al., 2016). Patients with chronic $\mathrm{HCV}$ are at risk of developing cirrhosis which may be followed by hepatocellular carcinoma (HCC),

\footnotetext{
* Corresponding Author

Eman Ahmed Awadallah Department of Clinical and Chemical Pathology, National Research Centre (NRC) Cairo, 12622 Egypt Phone: +2024106222 - 01223872147; Fax: +2023370931

E-mail: emanawad_28@yahoo.com
}

previous studies reports that about $94 \%$ of $\mathrm{HCC}$ patients are exposed with chronic HCV infection.HCC represents $13 \%$ of all cancers in Egypt (Schütte, 2015; Ziada, 2016). HCC is one of the most common neoplastic disorders worldwide (Forner and Bruix, 2012). In Egypt, it is the second common cancer among men and the sixth common cancer among women and represents the second cause of cancer related death (Bader El Din et al., 2016; Fiorino et al., 2016). It has very poor outcome because it is usually detected in advanced stage due to mild deviation of its clinical and pathological features from chronic liver disease (Sakamoto et al., 2010).

To date, serum biomarkers to diagnose early HCC have not been satisfactorily established. Alpha-fetoprotein (AFP) is widely used to detect primary HCC but its sensitivity and specificity are sub-optimal as in almost $80 \%$ of early HCC patients AFP levels is not elevated (Fiorino et al., 2016). 
Imaging techniques, including ultrasonography, Computed Tomography (CT) and Magnetic Resonance (MRI), have potentially limiting factors, including their availability, costs, and reproducibility (Khairy et al., 2016). Thus, early detection of HCC is still challenging and requires novel, sensitive and specific biomarkers that could detect tumor at an early stage where curative therapy can be applied (Sakamoto et al., 2010).

Different biological markers have been demonstrated to improve diagnosis of early HCC (Sakamoto et al., 2010). These markers could indicate development of HCC such as cyclaseassociated protein2 (CAP2) and macrophage migration inhibitory factor (MIF) (Sakamoto et al., 2010 and Zhao et al., 2011).In HCC, several studies demonstrated over-expression of CAP2 (Effendi et al., 2013 and Shibata et al., 2006 and Sakamoto et al., 2010).

However, the prognostic role of CAP2 in HCC remains elusive (Fu et al., 2015). As regard MIF different studies showed increased concentration of MIF in $\mathrm{HCC}$ and it also related to tumor size, tumor, node, and metastasis (TNM) staging and vascular invasion (Wang et al., 2014, and Zhao et al., 2011). However, results regarding the role of these factors in HCC are controversial.

MicroRNAs (miRNAs) are small non-coding RNAs that play key role in regulation of gene expression. Moreover, miRNAs are characterized by their remarkable stability in serum. Recent studies have reported that miRNAs regulate a variety of biological processes, including cell proliferation, differentiation and apoptosis (Huang et al., 2015). In addition, altered expression of miRNAs including miR -21 and miR-223 has been linked to the development of different human cancer but their role in HCC remains unclear (Khairy et al., 2016).

We hypothesized that levels of miR -21 and miR-223 in circulation could be altered in association with pathological changes in HCV patients' liver leading to HCC. If this hypothesis is established, major progress in HCC management could be achieved. The primary aim of this study was to assess expression of miR -21 and miR-223 in serum of HCC patients with HCV infection and correlate this expression with other clinical, laboratory features of $\mathrm{HCC}$ and other markers denoting stromal invasion and inflammation such as CAP-2 and MIF respectively.

\section{SUBJECTS AND METHODS}

\section{Subjects}

A total of 80 patients were recruited from the outpatient clinic of both the National Research Centre and the National Cancer Institute in Cairo from June 2015 to March 2016. All Patients were positive for HCV RNA testing. They were subdivided into 40 patients with newly diagnosed HCC at early stages (hepatic lesion mass of $2 \mathrm{~cm}$ or smaller as detected with four-phase CT and MRI) and 40 patients with Liver cirrhosis. HCC was diagnosed by presence of typical histological findings or imaging characteristics according to the Korean Liver Cancer Study Group guidelines and American Association for the Study of Liver Diseases (AASLD) guidelines (Lee et al., 2014). HCC staging was determined using the Barcelona Clinic Liver Cancer (BCLC) staging system (EASL, 2012). Liver cirrhosis was diagnosed by histological examination or clinical findings of portal hypertension (Marrero et al., 2009), and adequate imaging studies were repeatedly conducted on patients with liver cirrhosis to exclude HCC within 6 months of enrolment. Data of all subjects were obtained from medical records, pathology reports and personal interviews with the subjects. Exclusion criteria included: patients with positive HBsAg or with history of other causes of congenital liver diseases or chronic liver disease other than HCV, previous treatment for $\mathrm{HCC}$ or antiviral therapy for $\mathrm{HCV}$, extrahepatic dissemination, any associated malignancies other than HCC and any other systemic or chronic disease. The 40 control subjects enrolled in the study were seeking a routine health checkup in the National Research Centre clinics. These healthy subjects have met the following criteria: 1) seronegative for AFP; and 2) no evidence of any liver disease; 3) No evidence of associated malignancy, systemic or chronic disease. The protocol for this study was approved by National Research Centre Ethics Committee(ethical clearance number is 13167) and according to the ethical guidelines of the declaration of Helsinki with informed written consent taken from all participated individuals.

\section{Methodology}

All patients and controls were subjected to full clinical assessment and laboratory investigations including:

Clinical Examination: A detailed history taking including history of smoking, alcohol intake, occupational exposure to chemicals, drug intake, previous diseases etc. A thorough clinical assessment and total body examination with abdominal ultrasound and/or CT scan were done when indicated to detect tumor size and extension.

Laboratory Investigations: A blood sample was withdrawn from each subject and was divided, processed and stored according to the requirements and precautions of each test. The following investigations were done for all subjects of the study:

1. Conventional liver evaluation tests which include: aspartate aminotransferase (AST), alanine aminotransferase (ALT), total bilirubin, serum albumin and prothrombin time.

2. Estimation of AFP,was assessed by enzyme-linked immunosorbent assay technique (ELISA) following instructions on kits purchased from Immunospec Co.

3. Assessment of MIF and CAP2 was done using ELISA kit supplied by Glory Science Co., Ltd, USA (catalog number: 10078 and 15048 respectively).

\section{RNA analysis}

Extraction of miRNAs serum was done using miRNA assay Serum/Plasma Kit (Qiagen, Hilden, Germany). All serum RNA preparations were quantified by NanoDrop 1000 (Nanodrop, Wilmingtion, Delaware, USA). cDNA was done using TaqMan microRNA Reverse Transcription Kit (Applied Biosystems, 
Carlsbad, CA, USA) and microRNA-specific stem-loop primers (part of the TaqMan microRNA Assay Kit; Applied Biosystems). Real-time Quantitative PCR was performed using the Quantistudio12K flex Real-Time PCR System (Applied Biosystems), and the results were analyzed using the RQ manager software (Applied Biosystems).

The following formula $2-\Delta \mathrm{Ct}$ was used to calculate the miRNA levels in serum:

\section{$\Delta \mathrm{Ct}=$ mean $(\mathrm{Ct}$ of internal references $)-\mathrm{Ct}$ of target miRNA.}

The relative expression levels of miR-21 and miR-223 were calculated and normalized to miR-16 (Applied Biosystems, Foster City, CA) (Yu et al., 2015) using the comparative $\Delta \mathrm{Ct}$ method and the equation $2-\Delta \Delta \mathrm{CT}$, as described previously.

\section{Statistical analysis}

Analysis was performed using SPSS version 17 software for Windows. Data are reported as means \pm standard deviation (SD) or number (percentage). One-Way ANOVA analysis was used for multiple group comparisons. The least significant difference (LSD)-t test was used in pairwise comparison of averages among groups. Pearson's and Spearman's correlation tests ( $r=$ correlation coefficient) were used for correlating normal and non-parametric variables, respectively and linear regression analysis was done to confirm association of altered miRNA expression with HCC after adjustment of age and sex. A p-value of $<0.05$ was considered statistically significant. All tests were two-sided, and $\mathrm{P}<0.05$ was considered statistically significant.

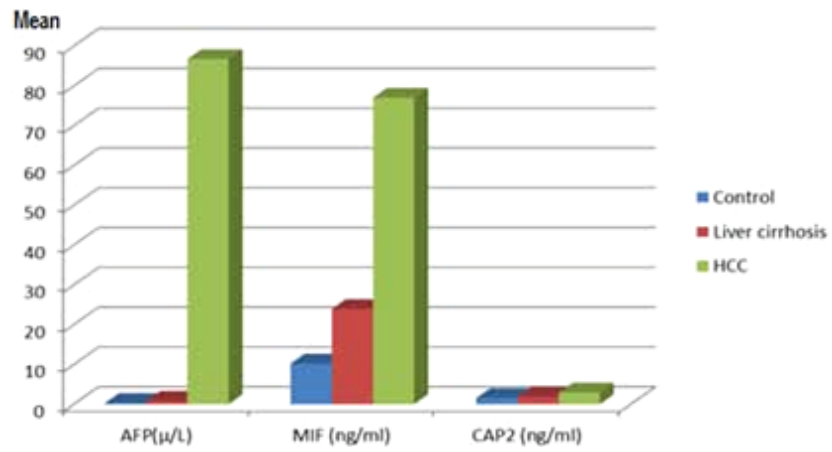

Fig. 1: Results of AFP, MIF and cap2 among studied groups. $\mathrm{AFP}=$ apha fetoprotein (represented in the chart as $10 \%$ of its real value) $\mathrm{HCC}=$ hepatocellular.
Clinical, demographic and laboratory data of the studied groups are shown in table 1. Our results showed increase of CAP2 serum levels in $\mathrm{HCC}$ compared to non $\mathrm{HCC}$ hepatic patients and controls $(0=0.001)$, while no significant differences were detected between HCC free hepatic patients and control group $(p=0.2)$. Serum levels of MIF increased in hepatic patients compared to controls $(p=0.001)$ and in patients with HCC compared to cirrhotic patients without HCC ( $\mathrm{p}=0.001)$ (Figure 1).

Levels of miR-21 were higher in patient groups compared to control groups $(\mathrm{p}<0.001)$ and in patients with HCC compared to HCC-free hepatic patients $(\mathrm{p}=0.002)$ (Table 1, Figure 2).

Serum levels of miR-223 showed no significant differences between $\mathrm{HCC}$-free hepatic patients and control group $(\mathrm{p}=0.2)$, while marked decrease in miR-223 expression levels was found in HCC patients compared to HCC-free hepatic patients and control group ( $\mathrm{p}=0.004,0.001$ respectively) (Table 1, Figure 2).

Linear regression analysis confirmed altered expression of miR-21 after adjustment of age and sex $(\mathrm{p}=0.01)$. No significant correlation was detected between miR-21 and age, sex, MIF, CAP2 and miR-223 ( $\mathrm{p}=0.2,0.9,0.9,0.8,0.8$ respectively).

Linear regression analysis confirmed altered expression of miR-223 in HCC patients compared to other patients after adjustment of age and sex $(\mathrm{p}=0.001)$. No significant correlation was detected between miR-223 and age, sex and CAP2 ( $\mathrm{p}=0.2$, $0.4,0.3$ respectively). Weak negative linear relationship was detected between miR-223 and MIF ( $\mathrm{r}=-0.25, \mathrm{p}=0.02)$.

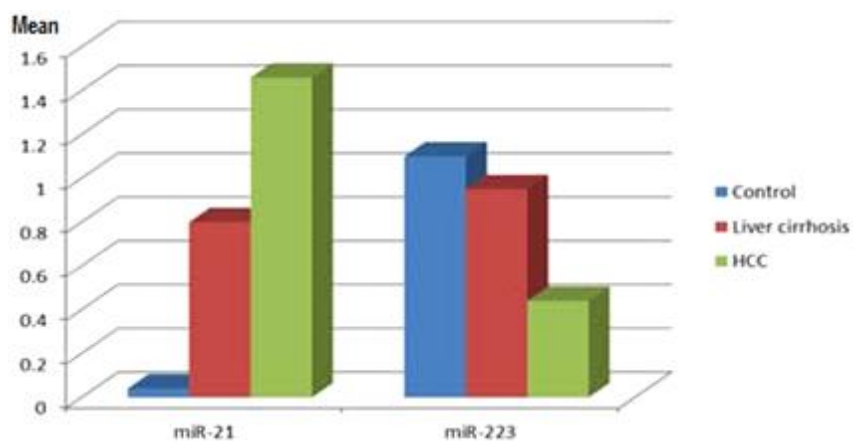

Fig. 2: Expression of miR -21 and miR- 223 among studied groups.

Table 1: Demographic clinical and laboratory data of studied groups.

\begin{tabular}{|c|c|c|c|c|}
\hline Variable & $\begin{array}{c}\text { Control } \\
(\mathrm{N}=40) \\
\text { Mean } \pm \text { SD }\end{array}$ & $\begin{array}{c}\text { Cirrhosis } \\
(\mathrm{N}=40) \\
\text { Mean } \pm \text { SD }\end{array}$ & $\begin{array}{c}\text { HCC } \\
(\mathrm{N}=40) \\
\text { Mean } \pm \text { SD }\end{array}$ & p value \\
\hline $\begin{array}{c}\text { Age (years) } \\
\text { Sex }\end{array}$ & $55.8 \pm 4$ & $58.3 \pm 7.3$ & $58.6 \pm 5.1$ & 0.3 \\
\hline Female & $17(42.5 \%)$ & $18(45 \%)$ & $11(27.5 \%)$ & \\
\hline AST (U/L) & $21 \pm 2.6$ & $62.8 \pm 31.1$ & $1673.8 \pm 1923$ & 0.001 \\
\hline ALT (U/L) & $22.9 \pm 3.1$ & $57.7 \pm 24.6$ & $1614.2 \pm 1760$ & 0.001 \\
\hline $\operatorname{AFP}(\mu \mathrm{g} / \mathrm{L})$ & $2.6 \pm 0.32$ & $8.4 \pm 8.8$ & $867.3 \pm 1618$ & 0.001 \\
\hline $\mathrm{MIF}(\mathrm{ng} / \mathrm{ml})$ & $10.2 \pm 2.8$ & $23.9 \pm 4.5$ & $77 \pm 27.4$ & 0.001 \\
\hline CAP2(ng/ml) & $1.5 \pm 0.2$ & $1.8 \pm 0.8$ & $2.91 \pm 0.9$ & 0.001 \\
\hline miR-223 & $1.1 \pm 1.06$ & $0.95 \pm 0.6$ & $0.44 \pm 0.4$ & 0.001 \\
\hline miR-21 & $0.04 \pm 0.05$ & $0.8 \pm 0.7$ & $1.46 \pm 1.3$ & 0.001 \\
\hline
\end{tabular}




\section{DISCUSSION}

Hepatocellular carcinoma (HCC) is one of the most frequently diagnosed cancers worldwide, and it is predominant in Asia and Africa (Fathy et al., 2016). Most HCC develops in patients with history of cirrhosis and chronic hepatitis as there is continuous inflammation and regeneration of hepatocytes (Gao et al., 2012).

Our results show deterioration in liver functions in patients groups when compared to control group. Our results agreed with Fathy and coworker (2016) who recorded that all measured liver functions as well as AFP showed highly significant difference between HCC patients and control group (Fathy et al., 2016). However, AFP is used in diagnosis of HCC with imaging techniques as ultrasonography, CT and MRI, whileFilmus and colleges (2004) found that the sensitivity of this marker is limited (41-65\%) (Filmus and Capurro, 2004).

Published data regarding human cancers reported differential expression of CAP-2. For example, CAP2 was upregulated in bladder and kidney human cancers and downregulated in breast fibroadenoma (Peche et al., 2007). Different studies reported over-expression of CAP-2 in HCC. Current published data suggest that CAP-2 is a potential diagnostic marker for stromal invasion in early HCC (Sakamoto, 2009 and $\mathrm{Fu}$ etal.,2015). Sakamoto demonstrated that CAP-2 was expressed in $70 \%$ to $100 \%$ of early HCC (Sakamoto, 2009). Our results showed increase of CAP-2 serum levels in HCC compared to non HCC hepatic patients, while no significant differences were detected between HCC free hepatic patients and control group, suggesting role of CAP2 in HCC pathogenesis and its role as potential diagnostic marker for early HCC. Consistent with our study, Shibata et al reported that CAP-2 expression was increased in early $\mathrm{HCC}$ and frequently increased with progression (Shibata et al., 2006).

Accumulating evidences indicate that MIF represents a cytokine linking inflammation and cancer, due to its key role in inflammation and cell proliferation (Bucala and Donnelly, 2007). With regard to HCC, most hepatic lesions produced by hepatitis B or $\mathrm{C}$ viruses develop chronic inflammation as well as abnormal cell proliferation due to cirrhosis. In our study, we found increased serum MIF levels in patients with HCC compared to cirrhotic patients without HCC. Our results suggest that circulating MIF is associated with the development of HCC and could be considered as a potential diagnostic marker for it. In agreement with our study, different studies reported increased levels of MIF in HCC (Zhao et al., 2011andSad et al., 2014). Zhao et al. reported that serum MIF may differentiate patients with HCC from individuals with other liver diseases, such as cirrhosis (Zhao et al., 2011). However, Ren et al. mentioned that MIF acts as a local autocrine factor rather than a systemic mediator in cancer, indicating that serum MIF alone was not a useful marker in clinical practice (Ren et al., 2005).

MiR-21 is described as one of the most important miRNAs contributed in cancer pathogenesis and progression.
Altered level of miR21 has been reported in different types of human cancer (Qi et al., 2011). This concept agrees with results of this work that showed up-regulation of miR-21 in hepatic patients compared to controls and in HCC patients compared to $\mathrm{HCV}$ patients. Our results agrees with other studies that reported overexpression of miR 21 in HCC and liver diseases (Connolly et al., 2008; Ladeiro et al., 2008). Connolly et al. demonstrated that upregulation of miR-21 occurs in precancerous stages of liver disease and in HCC in comparison with normal subjects (Connolly et al., 2008). Ladeiro et al. found that miR-21, were significantly overexpressed in patients with HCCs, associated with both viral- and non-viral risk factors (Ladeiro et al., 2008). Yan et al. reported in their meta- analysis, included five studies published from 2011 to 2014, that miR-21 over-expression in HCC correlated with poor outcome, and higher miR-21 expression was associated with metastases (Yan et al., 2015). However, published results regarding miR-21 showed high heterogeneity between studies. ElGuendy et al. reported downregulation of miR21 in liver biopsy of Egyptian patients with HCV Infection (El-Guendy et al., 2016). Other authors found lower levels of serum miR-21 in HBV positive patients with or without HCC than in healthy subjects (Qi et al., 2011). They mentioned that the detected circulating miRNAs might be derived from dying or active tumor cells. They added that miR-21 can act as either tumor suppressor or oncogene, depending on the targets they regulate and the associated epigenetic factors (Qi et al., 2011). The exact role of miR-21 in cancer is still under research and needs further studies in the future. This heterogeneity among studies is also mainly due to differences in reference control group (healthy subjects or patients with HBV or HCV -related liver disease), sample size and type, molecular techniques and the lack of common miRNA internal control.

MiR-223 is described as a bone marrow enriched miRNA that functions as regulator of cellular differentiation (Qi et al., 2011). Moreover, recent studies observed altered miR-223 expression in HCC, suggesting a potential role of this miRNA in pathology of liver disease (Roy et al., 2015; Roderburg and Luedd, 2014). In the current work, miR-223 was down regulated in hepatic patient group compared to control subjects and in patients with $\mathrm{HCC}$ compared to $\mathrm{HCV}$ positive patients. Our results agree with other studies reported decreased miR-223 expression in HCC. Khairy et al reported significant decrease in expression level in HCC group compared to non HCC groups (Khairy et al., 2016). Shi et al mentioned that miR-223 down regulated in HCC samples compared to matched non-tumor liver tissue (Shi et al., 2015). Bhattacharya et al found that miR-223, were expressed at significantly lower levels in the serum of HCC patients compared with patients with chronic liver disease and healthy subjects (Bhattacharya et al., 2016). On the other side, our results disagree with $\mathrm{Xu}$ et al who reported up regulation of miR-223 in HCC patients compared to controls and no significant difference was detected between HCC patients and patients with chronic hepatitis (Xu et al., 2011). Qi et al found that levels of miR-223 were significantly increased in HCC patients than in controls, while no 
significant differential expression was reported for this miRNA between HBV patients with and without HCC. Moreover, serum levels of miR-223 in HBV subjects without HCC were significantly higher than those in HCC patients or healthy subjects (Qi et al., 2011). Li et al also reported up regulation of miR-223 in $\mathrm{HBV}$ patients, while down regulation was reported in $\mathrm{HBV}$ negative HCC compared to HBV positive HCC (Li et al.,2010). The previous findings point out that elevated serum miR-223 in liver disease may come from tissue injury such as hepatitis. Thus, higher levels of miR-223 were reported in chronic hepatitis patients because chronic hepatitis may have more serious injury of hepatocytes than HCC patients (Qi et al., 2011). Discrepancy in published results regarding miR-233 in HCC may be explained by differences in pathology of associated liver diseases, sample type and criteria of selection, sample size, associated epigenetic factors.

Scientists identified a miRNA panel (miR-192, miR21,miR-36a, miR-223, miR-27a, miR-122 and miR-801) to discriminate healthy populations from patients with $\mathrm{HCC}$ as it provided high diagnostic accuracy (Zhu et al.,2013). This panel had considerable clinical value for the early diagnosis of HCC and could help patients who might have otherwise missed the curative treatment window benefit from optimal therapy (Giordano ,and Columbano, 2013). There are still some limitations in our study including small sample size and the fact that diagnosis of early stage HCC could be missed in some of cirrhosis patients which may partly lead to the false-negative of HCC diagnosis and bias in our results. In conclusion, altered serum miR-21 and -223 may play role in pathogenesis of $\mathrm{HCC}$ on top of $\mathrm{HCV}$ and liver cirrhosis. These miRNAs might serve as a novel and potential biomarker for detection of early $\mathrm{HCC}$ in $\mathrm{HCV}$ positive patients with liver cirrhosis. Large prospective studies are recommended before miR-21 and -223 can be used as a noninvasive tool for $\mathrm{HCC}$ screening in routine follow up of $\mathrm{HCV}$ patients.

\section{Financial support and sponsorship: Nil.}

Conflict of interest: The authors declare no conflict of interest.

\section{REFERENCES}

Atta MM, Atta HM, Gad MA, Rashed LA, Said EM, HassanienSel-S, Kaseb AO. Clinical significance of vascular endothelial growth factor in hepatitis $\mathrm{C}$ related hepatocellular carcinoma in Egyptian patients. J Hepatocell Carcinoma. 2016;3:19-24.

Bader El Din NG, Farouk S, El-Shenawy R, Ibrahim MK, Dawood RM, Elhady MM, Salem AM, Zayed N, Khairy A, and El Awady MK. Tumor necrosis factor- $\alpha-$ G308A polymorphism is associated with liver pathological changes in hepatitis $\mathrm{C}$ virus patients. World $\mathrm{J}$ Gastroenterol. 2016; 22(34):7767-7777.

Bhattacharya S, Steele R, Shrivastava S, Chakraborty S, Di Bisceglie AM, and Ray RB. Serum miR-30e and miR-223 as Novel Noninvasive Biomarkers for Hepatocellular Carcinoma.Am J Pathol. 2016; 186(2):242-247.

Bucala R and Donnelly SC. Macrophage migration inhibitory factor: a probable link between inflammation and cancer. Immunity 2007; 26: $281-285$.

Connolly E, Melegari M, Landgraf P, Tchaikovskaya T, Tennant BC, Slagle BL, Rogler LE, Zavolan M, Tuschl T, and Rogler CE.
Elevated expression of the miR-17-92 polycistron and miR-21 in hepadnavirus-associated hepatocellular carcinoma contributes to the malignant phenotype. Am J Pathol. 2008; 173: 856-864.

Effendi K, Yamazaki K, Mori T, Masugi Y, Makino S, and Sakamoto M. Involvement of hepatocellular carcinoma biomarker, cyclase-associated protein 2 in zebrafish body development and cancer progression.Exp Cell Res. 2013; 319(1): 35-44.

Elgharably A, Gomaa A I, Crossey M E, Norsworthy P J, Waked I, and Taylor-Robinson S D. Hepatitis C in Egypt - past, present, and future. Int J Gen Med. 2017; 10: 1-6.

El-Guendy NM, Helwa R, El-Halawany MS, Abdel Rahman AS, AlyTM, AlieldinHN, Fouad SA, Saeid H, and Abdel-Wahab AH. The Liver MicroRNA Expression Profiles Associated With Chronic Hepatitis C Virus (HCV) Genotype-4 Infection: A Preliminary Study. Hepat Mon. 2016; 16(4):e33881.

European Association for the Study of the Liver, European Organisation for Research Treatment of Cancer. EASL-EORTC clinical practice guidelines: management of hepatocellular carcinoma. J Hepatol 2012; 56(4): 908-943.

Fathy WM, Montaser BA, and El-Assal M.AngiotensinConverting Enzyme Gene Polymorphism (insertion/deletion) and The Risk of Hepatocellular Carcinoma in Egyptian HCV and HBV Patients.Journal of American Science, 2016;12(3): 116-124.

Filmus J, and Capurro M. Glypican-3 and alphafetoprotein as diagnostic tests for hepatocellular carcinoma.MolDiagn. 2004; 8(4): 207212.

Fiorino S, Bacchi-Reggiani ML, Visani M, Acquaviva G, Fornelli A, Masetti M, Tura A, Grizzi F, Zanello M, Mastrangelo L, Lombardi R, Di Tommaso L, Bondi A, Sabbatani S, Domanico A, Fabbri C, Leandri P, Pession A, Jovine E, and de Biase D. MicroRNAs as possible biomarkers for diagnosis and prognosis of hepatitis B- and Crelated-hepatocellular-carcinoma. World J Gastroenterol. 2016; 22(15): 3907-3936.

Forner $\mathrm{A}$ and Bruix $\mathbf{J}$.Biomarkers for early diagnosis of hepatocellular carcinoma. Lancet Oncol 2012; 13, 750-751

Fu J, Li M, Wu D, Liu L, Chen S, and Yun J. Increased Expression of CAP2 Indicates Poor Prognosis in Hepatocellular Carcinoma12. Transl Oncol; 8(5): 400-406.

Fu J, Li M, Wu DC, Liu LL, Chen SL, and Yun JP. Increased Expression of CAP2 Indicates Poor Prognosis in Hepatocellular Carcinoma. Transl Oncol. 2015; 8(5): 400-406.

GaoJ, Xie L, Yang WS, et al. Risk factors of hepatocellular carcinoma--current status and perspectives. Asian Pac J Cancer Prev. 2012; 13: 743-752.

Giordano S, and Columbano A. MicroRNAs: new tools for diagnosis, prognosis, and therapy in hepatocellular carcinoma. Hepatology. 2013; 57(2): 840-847.

Huang CS, Yu W, Cui H, Wang YJ, Zhang L, Han F, and Huang T. Increased expression of miR-21 predicts poor prognosis in patients with hepatocellular carcinoma. Int J Clin Exp Pathol. 2015; 8(6): 7234-7238.

Khairy A, Hamza I, Shaker O, Yosry A. Serum miRNA Panel in Egyptian Patients with Chronic Hepatitis C Related Hepatocellular Carcinoma.Asian Pac J Cancer Prev. 2016;17(5): 2699-2703.

Ladeiro Y, Couchy G, Balabaud C, Bioulac-Sage P, Pelletier L, Rebouissou S, and Zucman-Rossi J. MicroRNA profiling in hepatocellular tumors is associated with clinical features and oncogene/tumor suppressor gene mutations. Hepatology. 2008; 47: 1955-1963.

Lee JM, Park JW, and Choi BI. KLCSG-NCC Korea Practice Guidelines for the Management of Hepatocellular Carcinoma: HCC Diagnostic Algorithm. Dig Dis, 2014; 32(6): 764-777.

Li LM, Hu ZB, Zhou ZX, Chen X, Liu FY, Zhang JF, Shen HB, Zhang CY, and Zen K. Serum microRNA profiles serve as novel biomarkers for $\mathrm{HBV}$ infection and diagnosis of $\mathrm{HBV}$-positive hepatocarcinoma. Cancer Res. 2010; 70: 9798-9807.

Marrero JA, Feng Z, Wang Y, Nguyen MH, Befeler AS, Roberts LR, et al. Alpha-fetoprotein, des-gamma carboxyprothrombin, and lectin-bound alpha-fetoprotein in early hepatocellular carcinoma. Gastroenterology. 2009; 137(1): 110-118. 
Peche V, Shekar S, Leichter M, Korte H, Schroder R, Schleicher M, Holak TA, Clemen CS, Ramanath YB, and Pfitzer G. CAP2, cyclase-associated protein 2, is a dual compartment protein. Cell Mol Life Sci. 2007; 64 (19-20): 2702-2715.

Qi P, Cheng SQ, Wang H, Li N, Chen YF, and Gao CF. Serum microRNAs as biomarkers for hepatocellular carcinoma in Chinese patients with chronic hepatitis B virus infection. PLoS One. 2011; 6: e28486.

Ren Y, Law S, Huang X, Lee PY, Bacher M, Srivastava G, and Wong J. Macrophage migration inhibitory factor stimulates angiogenic factor expression and correlates with differentiation and lymph node status in patients with esophageal squamous cell carcinoma. Ann Surg, 2005; 242: 55-63.

Roderburg C, and LueddT.Circulating microRNAs as markers of liver inflammation, fibrosis and cancer. Journal of Hepatology, 2014; 61(6): 1434-1437.

Roy S, Benz F, Luedde T, and Roderburg C. The role of miRNAs in the regulation of inflammatory processes during hepatofibrogenesis. HepatobiliarySurgNutr. 2015; 4(1): 24-33.

Sad LAA, Younis SG, and Nagi HM (2014) Significance of Macrophage Migration Inhibitory Factor (MIF) and Anti P 53 Antibodiesin Prognosis of Hepatocellular Carcinoma. J Mol Biomark Diagn, 2014; 5:192. doi:10.4172/2155-9929.1000192

Sakamoto M, Effendi K, and Masugi Y. Molecular diagnosis of multistage hepatocarcinogenesis. Jpn J Clin Oncol. 2010; 40(9): 891-896.

Sakamoto M. Early HCC: diagnosis and molecular markers. J Gastroenterol. 2009; 44 (Suppl. 19): 108-111.

Sakamoto M1, Effendi K, Masugi Y. Molecular diagnosis of multistage hepatocarcinogenesis.Jpn J Clin Oncol. 2010; 40(9): 891-896.

Schütte K, Schulz C, Link A, and Malfertheiner P. Current biomarkers for hepatocellular carcinoma: Surveillance, diagnosis and prediction of prognosis .World J Hepatol. 2015; 7(2): 139-149.

Shi KQ, Lin Z, Chen XJ, Song M, Wang YQ, Cai YJ, Yang NB, Zheng MH, Dong JZ, Zhang L, and Chen YP. Hepatocellular carcinoma associated microRNA expression signature: integrated bioinformatics analysis, experimental validation and clinical significance. Oncotarget. 2015; 6(28): 25093-25108.

Shibata R, Mori T, Du W, Chuma M, Gotoh M, Shimazu M, Ueda M, Hirohashi S and Sakamoto M. Overexpression of cyclaseassociated protein 2 in multistage hepatocarcinogenesis. Clin Cancer Res. 2006; 12(18): 5363-5368.
Wang D, Luo L, Chen W, Chen LZ, Zeng WT, Li W, and Huang XH. Significance of the vascular endothelial growth factor and the macrophage migration inhibitory factor in the progression of hepatocellular carcinoma. Oncol Rep. 2014; 31(3):1199-1204.

Xu J, Wu C, Che X, Wang L, Yu D, Zhang T, Huang L, Li H, Tan W, Wang C, et al. Circulating microRNAs, miR-21, miR-122, and miR-223, in patients with hepatocellular carcinoma or chronic hepatitis. Mol Carcinog. 2011; 50: 136-142.

Yan SR, Liu ZJ, Yu S, and Bao YX. Investigation of the value of miR-21 in the diagnosis of early stage $\mathrm{HCC}$ and its prognosis: a metaanalysis. Genet Mol Res. 2015; 14(3): 11573-11586.

Yu F, Lu Z, Chen B, Dong P and Zheng J. MicroRNA-150: a promising novel biomarker for hepatitis B virus-related hepatocellular carcinoma .Diagnostic Pathology. 2015; 10:129.

Zhao YM, Wang L, Dai Z, Wang DD, Hei ZY, Zhang N, Fu XT, Wang XL, Zhang SC, Qin LX, Tang ZY, Zhou J, and Fan J. Validity of plasma macrophage migration inhibitory factor for diagnosis and prognosis of hepatocellular carcinoma. Int J Cancer. 2011; 129(10): 24632472 .

Zhu K, Da Z and Zhoul J. Biomarkers for hepatocellular carcinoma: progression in early diagnosis, prognosis, and personalized therapy. Biomarker Research, 2013; 1: 10

Ziada DH, El Sadany S, Soliman H, Abd-Elsalam S, Salama M, Hawash N, Selim A, Hamisa M, and Elsabagh HM. Prevalence of hepatocellular carcinoma in chronic hepatitis C patients in Mid Delta Egypt: A single center study. J Egypt Natl Canc Inst. 2016; 28(4): 257-62.

How to cite this article:

Elghoroury EA, Abdel Maksoud SA, Kandil D, El Kafoury MR, Hassan EM, Awadallah E, Hussein MA, Elghobary HA. Expression of microRNAs-21 and-223 in hepatocellular carcinoma on top of hepatitis $\mathrm{C}$ virus in a sample of Egyptian population. $\mathrm{J}$ App Pharm Sci, 2017; 7 (09): 052-057. 